\title{
Tuberculosis in China
}

\author{
Tian Hu, Wenjie Sun*
}

School of Public Health and Tropical Medicine, Tulane University, New Orleans, USA;

*Corresponding Author: wsun3@tulane.edu

Received 24 June 2013; revised 25 August 2013; accepted 2 September 2013

Copyright (c) 2013 Tian Hu, Wenjie Sun. This is an open access article distributed under the Creative Commons Attribution License, which permits unrestricted use, distribution, and reproduction in any medium, provided the original work is properly cited.

Tuberculosis (TB) has been and is still an important public health issue in China. According to the most recent report from the World Health Organization, China had an estimation of 1.4 million existing TB and 1 million incident TB in 2011 [1]. Among the new cases, approximately $1.2 \%$ combined the existing HIV infection [1]. Since TB is directly related to people's health status and work capability, therefore, the control of TB prevalence has been of priority both in the public health and in the economic development of China.

The progress in controlling TB in China is, however, currently influenced by the following two major factors. First, the migration and health system compounds the prevention from TB. It is known that eighty percent of TB exists in rural areas, particularly in north and northwestern regions with low socioeconomic status in China. In the past three decades, there has been an increasing number of people from these areas that immigrated to the large urban cities; and they could potentially result in the spread of the disease in cities if they were TB-carriers. The urban living style and working conditions promote the transmission of TB in the big cities via crowded conditions and airborne pollution. However, the subsidized management of TB is only available through facilities in the birth place of immigrants and it is sometimes difficult for them to get screened, diagnosed and treated in the big cities.

Also, multidrug resistant (MDR) TB has been another pressing public health issue in China. MDR TBs are spread from individual to individual in the same manner as regular TB, but are usually resistant to at least isoniazid and rifampicin, the two most powerful first-line anti-TB medications. Since most TB therapy consists of short-course chemotherapy, the treatment could only cure a small proportion of MDR TBs and the treatment using second-line TB medications is usually very expensive. Therefore, MDR TBs have brought about difficulty in the treatment, especially in developing countries such as China. With an increasing number of patients not being treated, TB would be problematic, and the epidemic of TB would be revived. In 2013, the prevalence of MDR TB in mainland China was $5.3 \%$ to $27.4 \%$ [2].

Despite these challenges, Chinese government has successfully addressed TB problem in the past few decades, and has great contribution to global TB prevention. Also, Centers for Disease Control in China together with other institutes conducts many preventive interventions of TB funded by The Global Fund to Fight AIDS, Tuberculosis, and Malaria program initiated by the World Health Organization. Chinese health professionals believe that they could fulfill the world-wide goal towards elimination of tuberculosis by 2050 [3].

\section{REFERENCES}

[1] WHO (2012) Global Tuberculosis Report 2012, WHO.

[2] Yang, Y., Li, X.W., Zhou, F., Jin, Q. and Gao, L. (2011) Prevalence of drug-resistant tuberculosis in mainland China: Systematic review and meta-analysis. PloS One, 6, e20343. doi:10.1371/journal.pone.0020343

[3] Jassal, M.S. and Bishai, W.R. (2010) Epidemiology and challenges to the elimination of global tuberculosis. Clinical Infectious Diseases: An Official Publication of the Infectious Diseases Society of America, 50, S156-S164. 